\title{
A Passive UHF RFID System over Ethernet Cable for Long Range Detection
}

\author{
Zhe Fu ${ }^{1}$, Ian H. White ${ }^{2}$, Richard V. Penty ${ }^{1}$ and Michael J. Crisp ${ }^{1}$ \\ ${ }^{1}$ Centre for Photonic Systems, Electrical Division, Department of Engineering \\ University of Cambridge, 9 JJ Thomson Avenue, Cambridge, CB3 0FA, UK \\ ${ }^{2}$ Vice Chancellor's Office, University of Bath, Claverton Down, Bath BA2 7AY, UK
}

\begin{abstract}
This paper proposes a new form of passive UHF RFID system which has high tag detection accuracy but lower costs than existing systems for wide-range RFID scenarios requiring greater flexibility. This new system concept consists of a central baseband controller and a remote antenna subsystem, connected using a twisted-pair cable. Baseband signals are transmitted over the twisted-pair cable during the inventory session, and the transmitted radio frequency (RF) signals are up and down converted in the antenna subsystem. $-88 \mathrm{dBm}$ reader sensitivity is achieved with an active leakage cancellation block, showing little degradation in tag detection performance over a $300 \mathrm{~m}$ of Cat5e cable between the controller and the antenna. An average leakage suppression of $36.9 \mathrm{~dB}$ can be achieved with a fixed transmission power of $26.5 \mathrm{dBm}$. Compared with conventional RFID systems using coaxial cables between the reader and antenna, the presented system is superior in terms of link distance, link cost, and installation flexibility.
\end{abstract}

Keywords-RFID; twisted-pair cable; cat5e; long distance; deployment; flexibility; low cost;

\section{INTRODUCTION}

Radio frequency identification (RFID) is an attractive technology to potentially supersede barcode labels in retail and the manufacturing industry. This is because items can be detected without human intervention or line of sight. However the high cost of the hardware, limited read range, and uncertain detection reliability are considerable barriers to many potential applications. These challenges present great difficulties for wide-area RFID applications. Other issues such as deployment difficulty and system maintenance have also created barriers to untrained users. As a result, there have been few rollouts of wide-area RFID technology.

Recently, there has been increased interest in RFID systems which address these challenges. Some low-cost handheld RFID readers have been designed with lower cost and high detection rate, but they still require intensive manual input in order to read all items over a wide area [1]. For a conventional fixed RFID reader system, multiple readers must be deployed to cover the required area. For instance, 80 readers (each likely to require multiple antennas) are deployed over 4 levels in a building [2] and massive numbers of readers are required to track miners and equipment in underground mining service applications [3]. In order to reduce the

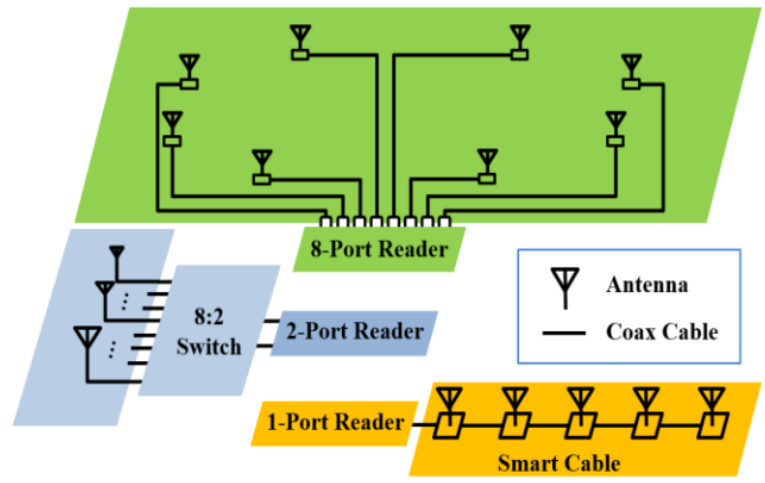

Fig. 1. Published multiantenna solutions for single RFID reader [4-7]

hardware cost, novel multi-antenna RFID systems have been developed. This kind of RFID reader significantly improves the coverage and diversity of a single RFID reader (Fig. 1). Buendia et al. [4] introduced a smart cable for a single reader RFID system, which allowed their system to cover an area of $16 \mathrm{~m} \times 2.5 \mathrm{~m}$ along a corridor. In this smart cable, single-poledouble-throw (SPDT) switches and Wilkinson power combiners are applied to feed 5 rectangular patch antennas in a time division multiplexed approach. A similar solution was proposed by Rodas et al. [5]. Here, they designed a Powerover-Ethernet (PoE) supported reader-plus-switch to extend a 2-port RFID reader to 8 ports or a 4-port RFID reader to 16 ports. The extended antenna ports are automatically switched based on the pre-programmed software so that the system can detect over a wide area without tag-to-reader and reader-toreader interferences. A multiport RFID reader, which can further reduce the cost of extra antenna control units, has been designed by Wang et al. [6]. By adding frequency and phase hopping techniques, along with the antenna diversity the RFID system performance can be enhanced [7]. The demonstrated system can achieve near $100 \%$ tag detection rate over a $20 \mathrm{~m}$ $\times 15 \mathrm{~m}$ area, a substantial increase over conventional switched multi-antenna RFID systems. The increase in detection rate, can also be viewed as allowing a potential reduction in the required number of readers or antennas to achieve a particular detection rate when compared to a more conventional system. 
A key requirement of the system is the coherence of the RF signals at multiple Tx antennas.

For the above outlined RFID systems, many coaxial cables are required to connect the antennas to the RFID reader and other elements. Since coaxial cables suffer high attenuation at around $900 \mathrm{MHz}$, the RFID systems discussed above are limited in the cable lengths they can support. Additionally, the heavy weight and limited bend radius of coaxial cables increases the installation difficulty in many scenarios. Coxial cables are also more expensive than other types such as twisted pair. Thus, this configuration is only suitable for experimental and small area applications. However new connection approaches for multi-antenna systems which scale more effectively are still required to provide RFID coverage over very large areas. In this paper, a novel long-range passive UHF RFID system over a twisted-pair Ethernet cable is proposed. This new system consists of a central controller and a remote antenna subsystem, connected by a commonly-used Ethernet cable such as Category 5e (Cat5e). This allows long cable lengths which enable high deployment flexibility and cost benefits, as well as the possibility of new architectures whereby multiple distributed antennas can be cascaded off a single cable to reduce installation costs.

The remainder of the paper is organized as follows. In Section II, we introduce the new reader architecture with a full explanation of each operational block. Following that, the performance of the designed system is shown in Section III. A comparison study of this new system and other off-the-shelf RFID applications is provided in Section IV. Finally conclusions are presented in Section V.

\section{SYSTEM DESIGN}

In a conventional RFID system, both the baseband block and radio frequency (RF) block (consisting of the RF front end and a frequency synthesizer) are integrated on a single PCB (or even IC) to form an RFID reader, with a short coaxial cable connecting the reader to its antenna (see Fig. 2(a)). Since the cable loss is directly proportional to the cable length and is normally of the order of dB's per meter at frequencies around $900 \mathrm{MHz}$, the reader's maximum output power and antenna gain limit the maximum allowable cable length if the maximum effective isotropic radiated power (EIRP) allowed by the local regulations is to be reached. For a typical $1 \mathrm{~W}$ conducted power and $6 \mathrm{dBi}$ gain antenna, this is often only a few metres. While the maximum cable length in the downlink can be increased by increasing the conducted output power (at the expense of electrical power consumption), in the uplink the cable loss eventually results in a noise-limited link and reduced sensitivity. Simply adding an amplifier after the receiver antenna to compensate the cable loss may also lead to serious leakage problems since it is inherent in RFID systems that some of the transmitted carrier power will leak into the front end of the receiver. Additional gain in the receiver to overcome cable loss must handle the level of leakage without causing compression. This is because RFID transmission and reception both operate at the same frequency and time, and therefore a strong transmission signal is not only received by the passive tags but also by the reader receiver. The highpower transmission signal, which leaks into the receiver chain,

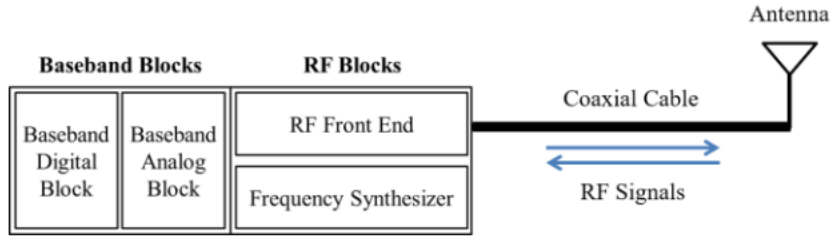

(a) Conventional RFID System

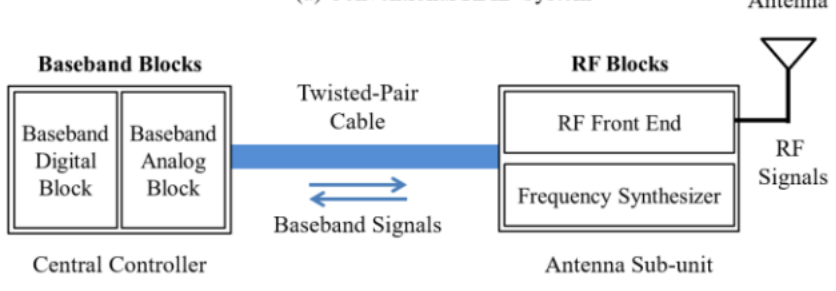

(b) Proposed New RFID System

Fig. 2. A comparison of a conventional RFID system (a) and the proposed RFID system (b)

increases the noise floor and may cause saturation of the amplifiers and analog to digital converters.

In a monostatic configuration, the leakage arises from imperfect isolation of the circulator or directional coupler combined with a mismatch in antenna impedance. In a bistatic configuration, the main source of the leakage is coupling between the two antennas. Although a bistatic system offers better performance in terms of leakage effects, its higher cost, additional size, and lower design flexibility are resulting issues that cannot be avoided. Reflection, caused by the surrounding objects, is another source of leakage which may be time varying. For a multi-antenna RFID system, the leakage problem becomes serious and complex due to the increasing number of leakage sources although those which are further away will be attenuated more. For both monostatic and bistatic systems, leakage suppression blocks have been shown to reduce the detrimental effects.

Twisted-pair cable such as Category 5e (Cat5e) is an attractive option for a new configuration of RFID system to extend antenna deployment distance due to the low cost and ease of installation and termination compared to a coaxial cable. However, Cat5e cable suffers very high attenuation in the UHF frequency band $(860-960 \mathrm{MHz})$. Nonetheless, this problem can be solved by splitting the functionality of the RFID reader between a central controller and an antenna unit, such that only baseband signals need to be carried between the two. The structure of this new system is shown in Fig. 2(b). In the forward link, the baseband transmission signals are generated in the digital block according to the air interface standard (e.g. Class 1 Gen 2). Following digital to analog conversion, the analog baseband signals are transmitted to the remote antenna subsystem over a twisted-pair cable. Upconversion is performed in the RF front end at the remote antenna subsystem. Antennas can be tightly integrated with the subsystem or connected by a short coaxial cable depending on the requirements of the installation. In the reverse link, the backscattered signals experience similar processes but in 


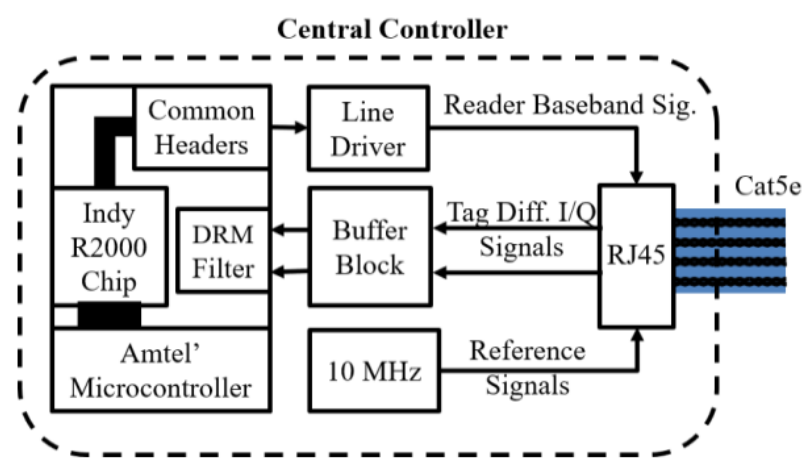

Fig. 3. Block diagram of the central controller

reverse order. RF signals are received at the antenna, and mixed back to I and Q baseband components in the antenna subsystem before transmission over the two pairs of the Cat5e cable to the central controller where digitization and protocol operations are executed. The shared local oscillator for up and down conversion is generated in the antenna subsystem, using a reference tone provided by the central controller, such that multiple antenna subsystems are phase locked allowing methods described in Section I which require coherent antenna to be used.

\section{A. Central Controller}

The high-level block diagram of the central controller is shown in Fig. 3. This design is based around the Impinj Indy R2000 chip [8] which provides all the baseband and RF functions required to implement a reader based on the EPC global Gen 2/ISO 18000-6C standard (although in this implementation some of the functionality is unused). An Atmel AT91SAM7S256 [9], microcontroller implements the EPC Class 1 Gen 2 protocol and provides an interface for external control. The baseband transmission signal can be accessed from the analog test pins of the R2000 as a differential signal. However, since these baseband signals are directly connected to the internal analog bus, they have little current driving capability. A line driver is therefore designed to amplify and buffer these signals without loading down the signal bus. Due to the ASK modulation in the downlink, the data on the I channel is null (0) and signals only exist on the $\mathrm{Q}$ channel. In this way, only a single twisted pair is required to transmit the downlink signals.

In respect of the uplink, an analog buffer block in the central controller is employed to buffer the received baseband signals and provide noise filtering. After this block, the analog tag signals are fed into the R2000 chip for additional filtering and processing via the inputs designed for the external dense reader mode (DRM) filters, thus bypassing the RF to IF conversion stages on the IC. The reference signal block provides a $10 \mathrm{MHz}$ reference signal for the frequency synthesizer in the antenna subsystem to allow multiple antenna units to operate coherently.

\section{B. Antenna Subsystem}

In previous work by $\mathrm{Fu}$ et al. [10], the preliminary antenna subsystem was mainly composed of modulator and

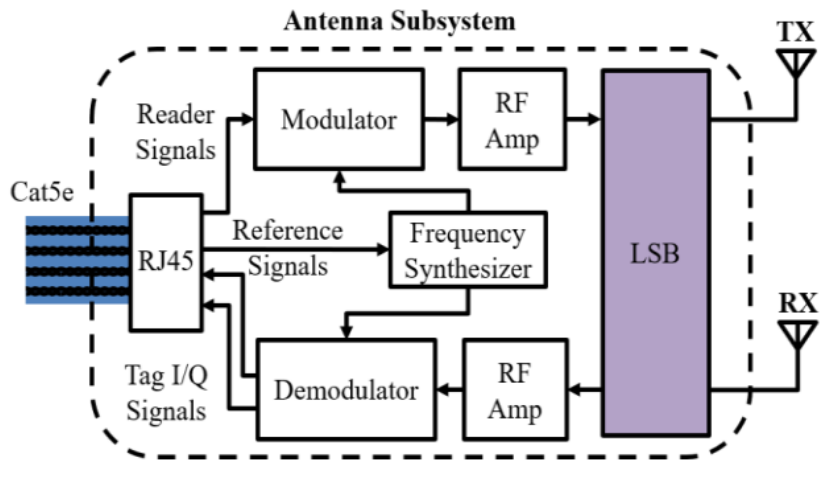

Fig. 4. Block diagram of antenna subsystem

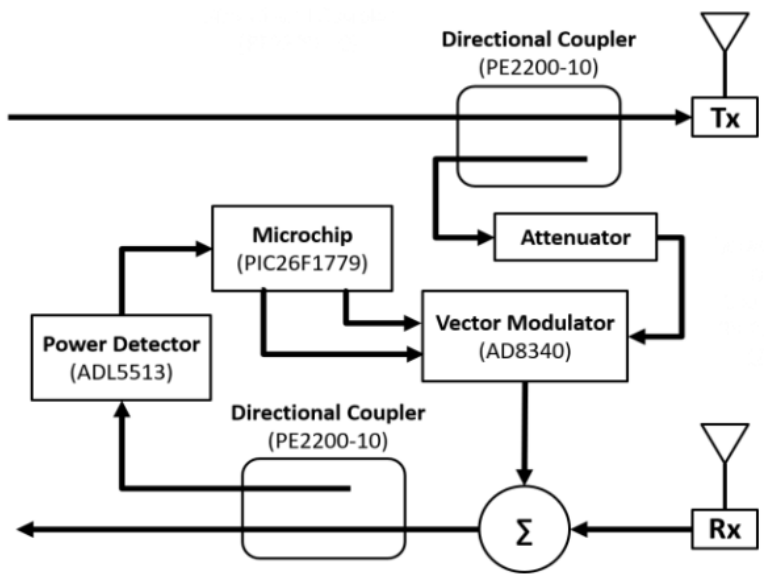

Fig. 5. Block diagram of leakage canceller block

demodulator, RF power amplifiers, and a PLL-based frequency synthesizer (see Fig. 4). The AD8349 [11] modulator and the ADL5380 [12] demodulator were used to fulfil up- and down-conversion tasks. The carrier frequency was generated by a fractional-N/integer-N PLL-based frequency synthesizer ADF4350 [13], which can work with either an internal oscillator or an external reference source. In this implementation, an external $10 \mathrm{MHz}$ reference signal from the central controller is applied. An RF amplifier (RF5110G [14]) and a low noise amplifier (ZX60-0916LN+ [15]) are adopted to provide sufficient gain for the signals both in the downlink and uplink. The output power of the proposed system can be controlled from $-10 \mathrm{dBm}$ to $+35 \mathrm{dBm}$.

The preliminary antenna subsystem is designed for a bistatic operation. It requires the transmission and reception antennas to be deployed in a way that ensures a minimum isolation. In order to increase the system reliability and address the incoming leakage from structural reflections and imperfect antenna isolation, a leakage canceller block (LCB) is added to the subsystem. Fig. 5 presents the block diagram of this leakage canceller, which applies a phase and amplitude control approach to solve the leakage problems. The core principle of this approach is to generate an identical signal in anti-phase having the same amplitude as the leakage signal, such that when superimposed on the receiver signal 
destructive interference occurs leaving only the backscattered tag signal. Several other leakage cancellation studies have utilized this methodology for RFID system [16-18].

The subsystem implementation employs, an AD8340 [19] vector modulator from the Analog Devices Company to vary both the phase and amplitude of the cancellation signal. The gain of this modulator can be set between $-2 \mathrm{~dB}$ and $-32 \mathrm{~dB}$, and the phase can be shifted over an entire cycle $\left(0-360^{\circ}\right)$. An ADL5513 [20] power detector is used to measure the residual leakage level. According to the specifications, its dynamic range in the RFID frequency band is from $-62 \mathrm{dBm}$ to $8 \mathrm{dBm}$. The voltage-power slope of this power detector is $21 \mathrm{mV} / \mathrm{dB}$. The detection error is less than $0.5 \mathrm{~dB}$ when the input RF power is in the proper range. Since at least two DACs and one ADC are needed to generate two control signals and receive one feedback signal respectively, a PIC16F1779 microcontroller [21] is selected. The critical task for this microcontroller is to quickly find the optimal control value. The details of the applied algorithm are provided in Section III Subsection B. In terms of the rest of the RF components, a $3-\mathrm{dB}$ power combiner is applied to add the canceller signal to the received signal. Two 10-dB directional couplers are used to tap the transmission signal to be used in cancellation and the residual power in the received signal after cancellation. An additional attenuator is used to protect the vector modulator from the high-power downlink signal.

\section{Twisted-pair Cable}

Category-5e cable is selected for building this new system, since it has sufficient bandwidth $(100 \mathrm{MHz})$ and its cost is relatively low compared to other higher-bandwidth twistedpair cables as well as having a large installed base. There are four twisted pairs in one Cat5e cable (Fig. 6), and all pairs are used in this new system; one for transmitting the baseband reader signals, one for the synthesizer reference frequency from reader controller and two for receiving in-phase and quadrature down-converted tag signals from the antenna subsystem. Although not implemented for this proof of concept demonstration, it would be possible to transmit the DC power required for the antenna subsystem over the pairs in a manner similar to power over Ethernet (PoE). Since these Ethernet cables have the same wiring scheme, other types of twisted-pair cable such as Cat6 can also be applied to the

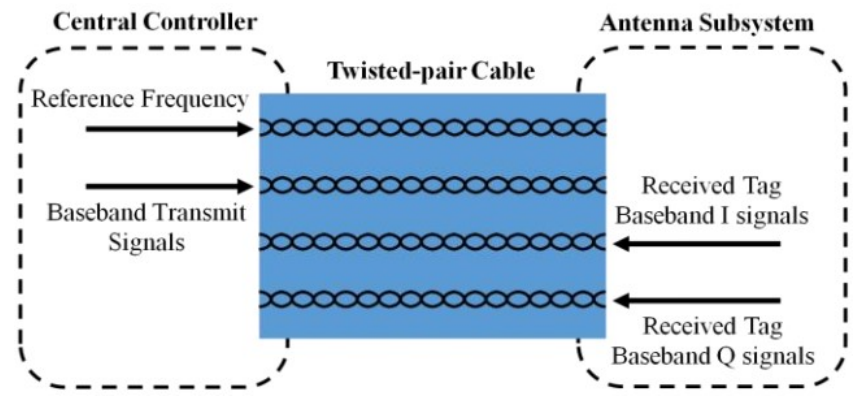

Fig. 6. Signals in the Cat5e Ethernet cable controller-subsystem connection. It would also be possible to use fewer pairs by multiplexing signals having substantially different frequencies.

In addition to the attenuation benefits of the Cat5e scheme it is also worth noting that it is lower cost, and easier to install than LMR-400 and LMR-1700 co-axial cables commonly used for RFID applications, owing to the narrower diameter, smaller minimum bend radius, and easy termination. More details of comparison in terms of cable loss, weight and price are shown in Table I.

TABLE I. COMPARISONS OF CAT5E CABLE AND COAXIAL CABLES

\begin{tabular}{|c|c|c|c|c|}
\hline \multicolumn{2}{|l|}{ Cable Type } & $\begin{array}{l}\text { Cat5e } \\
\text { Cable }\end{array}$ & $\begin{array}{c}\text { LMR-400 } \\
\text { Coaxial Cable } \\
{[22]}\end{array}$ & $\begin{array}{c}\text { LMR-1700 } \\
\text { Coaxial Cable } \\
{[23]}\end{array}$ \\
\hline \multicolumn{2}{|c|}{ Testing Frequency } & $350 \mathrm{kHz}$ & $900 \mathrm{MHz}$ & $900 \mathrm{MHz}$ \\
\hline \multirow{3}{*}{ Cable Loss (dB) } & $30 \mathrm{~m}$ & 0.32 & 3.84 & 0.93 \\
\hline & $90 \mathrm{~m}$ & 0.96 & 11.52 & 2.79 \\
\hline & $150 \mathrm{~m}$ & 1.60 & 19.2 & 4.65 \\
\hline \multirow{2}{*}{ Weight (kg) } & $30 \mathrm{~m}$ & 0.93 & 3 & 13 \\
\hline & $150 \mathrm{~m}$ & 4.65 & 15 & 165 \\
\hline \multirow{2}{*}{$\begin{array}{l}\text { Approximate Price } \\
(£)\end{array}$} & $30 \mathrm{~m}$ & 24 & 90.9 & 1,306 \\
\hline & $150 \mathrm{~m}$ & 120 & 454.5 & 6,530 \\
\hline
\end{tabular}

\section{IMPLEMENTATION AND RESULTS}

\section{A. System Performance without $L C B$}

In order to fully and reliably evaluate this new RFID system performance, an RFID Tester TC-2600A [24] is used. This tester can emulate a reference RFID tag to measure reader performance in terms of sensitivity. The sensitivity measurement is performed by calculating the reader's bit error rate (BER) and frame error rate (FER) by comparing its sent RN16 ACK with the known RN16 generated by the Tester (thus assuming that all errors occur in the readers demodulation of the RN16). The system sensitivity is defined by setting a threshold BER or FER and finding the minimum backscatter power where this can be achieved.

A number of tests were conducted to measure the system sensitivity with various lengths of Cat5e cable. In these tests, Miller-2 PR-ASK modulation was used for the emulated air interface, and a $10 \mathrm{MHz}$ reference signal with a power of 10 $\mathrm{dBm}$ was transmitted from the central controller over the Cat5e cable. Due to the maximum input power limits of the RFID Tester, the output power of the antenna subsystem was set to $+16.4 \mathrm{dBm}$ at a carrier frequency of $867.4 \mathrm{MHz}$. The threshold of FER for determining the sensitivity was set at $45 \%$. The system sensitivity for each cable length was measured 10 times with the average sensitivity value shown in Fig. 7. It was determined that the best sensitivity result of this system was $-94.5 \mathrm{dBm}$ when $30 \mathrm{~m}$ Cat5e cable was used to connect the controller and antenna subsystem. After increasing the length of Cat5e cable to $150 \mathrm{~m}$, the sensitivity reduced slightly to $-94.2 \mathrm{dBm}$. As a result, the system sensitivity is initially independent of cable length up to $150 \mathrm{~m}$. It is worth noting, however, that the RFID Tester gives excellent isolation 
between the $\mathrm{Tx}$ and $\mathrm{Rx}(\sim 90 \mathrm{~dB})$; in practice, the achieved isolation and hence sensitivity is likely to be lower.

In order to investigate the dependence of sensitivity on the cable attenuation, the attenuation of backscattered signal in the Ethernet cable was measured (although for experimental reasons, this was emulated using a function generator at 350 $\mathrm{kHz}$ ). The results are summarized in Table I alongside the losses of common coax cables derived from their specifications. It can be seen that baseband signals suffered $0.3 \mathrm{~dB}$ attenuation over $30 \mathrm{~m}$ and $1.6 \mathrm{~dB}$ over $150 \mathrm{~m}$ lengths of Cat5e cables respectively. The attenuation difference between these two lengths is $1.3 \mathrm{~dB}$, which is significantly larger than the sensitivity reduction $(0.3 \mathrm{~dB})$. Therefore, the reader sensitivity is not entirely due to the cable loss.

A practical demonstration of the detection range of a 300 m Cat5e connected RFID system was carried out (Fig. 8). Two 8.5 dBic circularly polarized antennas were deployed in height of $1.5 \mathrm{~m}$ connected to an antenna subsystem in a bistatic configuration (Fig. 8) using two $2 \mathrm{~m}$ coaxial cables. A UPM Raflatac DogBone tag [25] could be successfully detected at the same height $6 \mathrm{~m}$ away from the antennas with a downlink

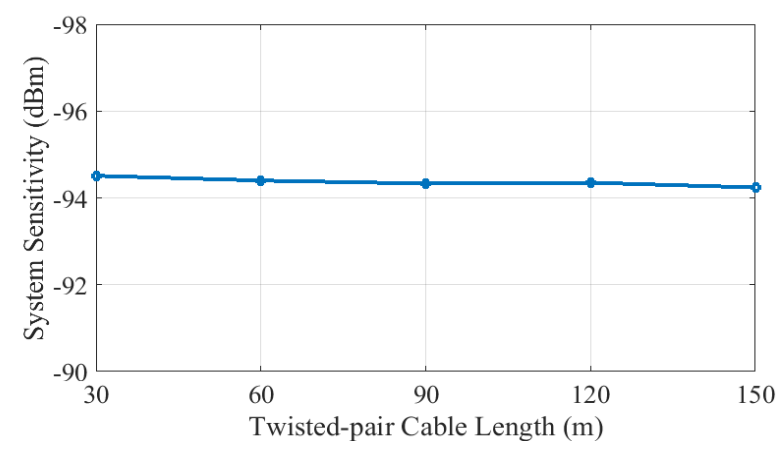

Fig. 7. Sensitivity performance over different cable lengths

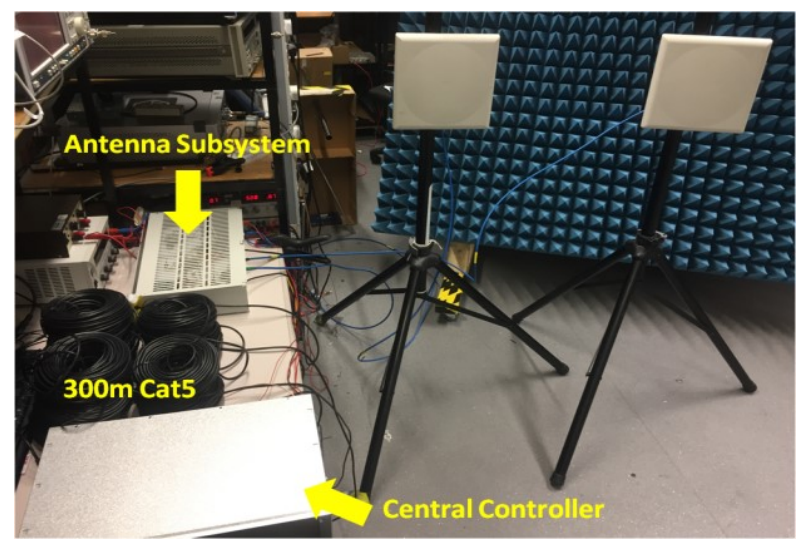

Fig. 8. Detection range measurement setup transmission power of $31.9 \mathrm{dBm}$ EIRP. Here the transmission distance is limited by the space available and is not an upper limit. However, it is useful to demonstrate the potential for long-range tag reading over a Cat5e cable.

\section{B. System Performance with $L C B$}

The leakage canceller block helps the system to address the leakage effects, enabling monostatic operation. Since the phase and amplitude control method is applied in the LCB, the most straightforward way to find the optimum parameters for setting the phase and amplitude is an exhaustive search algorithm to find the global minimum. In this algorithm, all the possible combinations of the selectable parameters are tested. When the scanning step size of the control parameters is one, the total number of tests is equal to the number of possible combinations. For example, a leakage canceller using the phase and gain control method must have $\mathrm{N}$ and $\mathrm{M}$ settings for the phase and gain respectively. Then the total number of measurements is $\mathrm{N} \times \mathrm{M}$.

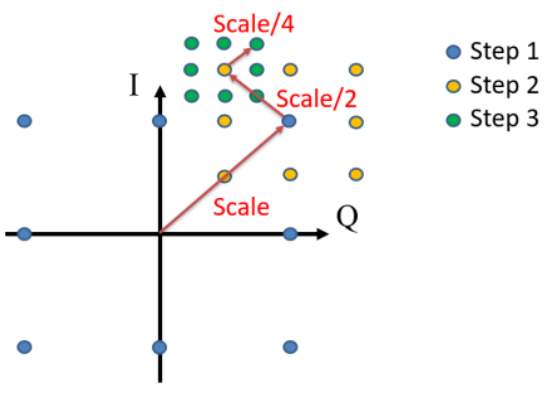

(a) Example of scale change in steps

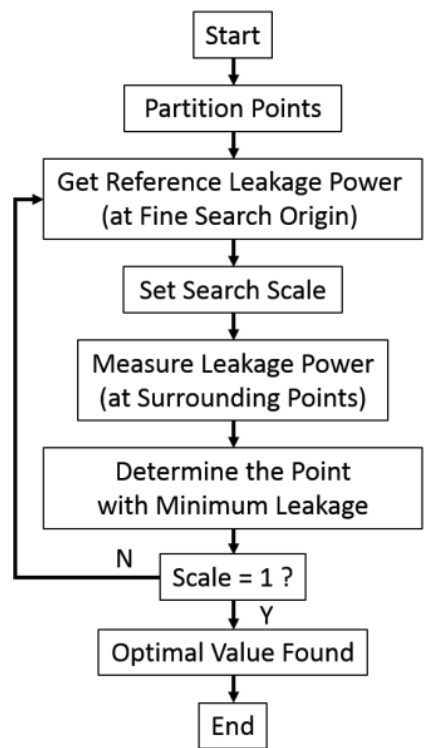

(b) General processes of the improved algorithm

Fig. 9. Improved full search algorithm for LCB 
This algorithm is extremely time-consuming. Both the periods for parameter initialization $\left(\mathrm{T}_{\mathrm{i}}\right)$ and residual leakage measurement $\left(\mathrm{T}_{\mathrm{s}}\right)$ have to be considered. Accordingly, scanning all the combinations requires an overall time of $\left(\mathrm{T}_{i}+\right.$ $\left.\mathrm{T}_{\mathrm{s}}\right) \times \mathrm{N} \times \mathrm{M}$. This low-efficiency search algorithm will cause serious problems if the time for self-cancellation measurement becomes longer than the maximum dwell time on a particular frequency, which is limited in some geographies by radio regulations. Therefore, improved search algorithms have been developed to reduce the optimisation time. Among these improved algorithms, a popular one which is applied in many commercial RFID readers and chips uses hierarchical grid partitioning to find the best point [8].

Rather than measuring all the points, the applied searching algorithm in this LCB needs to measure fewer points to find the optimal control value for the leakage suppression system. Similar to the example shown in Fig. 9(a), the first step of the algorithm is to symmetrically divide all the points into four sectors and then to measure the central point in each sector. The second step is to determine the Point $O$ that offers the minimum leakage component and set the initial value of the search scale (known also as convergence rate). The initial value of the search scale is usually determined by the resolution of the DACs, ADCs, and Power Detector in the leakage canceller block. For example, if the LCB can only support a resolution of 128 power levels, the scale can be initialized at a value of 64. After this step, the leakage measurement moves to the surrounding points of the Point $\mathrm{O}$ with half of the scale such as 32 . This process is repeated until the scale reduces to 1 , and finally the optimal point can be obtained. It can be found that the scales in some searching steps are non-integer values. In order to obtain a quantized value for $\mathrm{ADC}$ and $\mathrm{DAC}$ to find the related point and also avoid missing points, the floating scale in the algorithm is rounded to the next largest integer. The general processes of this improved search algorithm is expressed in Fig. 9(b).

Since our implementation of the proposed new system operates in the bistatic antenna configuration, the leakage level is largely dependent on the distance between the transmission and reception antennas. In order to reduce the environmental effects, the measurement at this stage was conducted in a static environment with no objects moving during the testing. The Tx and Rx antennas were deployed toward the same direction with a gap of $1.5 \mathrm{~m}$. The operation frequency is equal to 867 $\mathrm{MHz}$, and the transmitted power is $26.5 \mathrm{dBm}$. An additional directional coupler is used to measure the received signal after cancellation using a spectrum analyzer. According to the measurement, the system receiver suffered from an average -8 $\mathrm{dBm}$ leakage signal during tag detection. This leakage level is many orders of magnitude stronger than the received tag power. However, when the leakage suppression block has completed the self optimisation algorithm outlined above, the leakage level decreases to $-43 \mathrm{dBm}$ within $40 \mathrm{~ms}$, and the strength of tag signals can be clearly seen in the frequency domain (Fig. 10). In order to obtain a reliable suppression effect result of this leakage canceller, 20 similar tests were undertaken and the average result (plotted in Fig. 10) for average leakage suppression effect is $36.9 \mathrm{~dB}$.

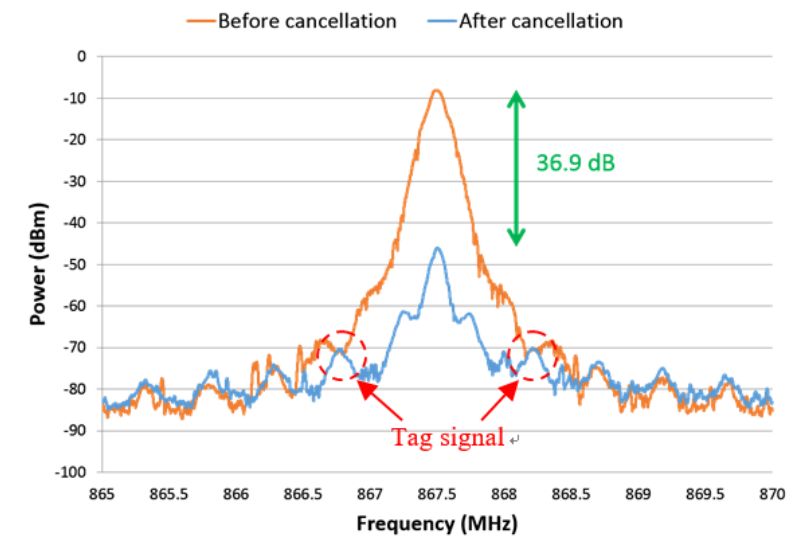

Fig. 10. Average leakage suppression effect of the proposed canceller

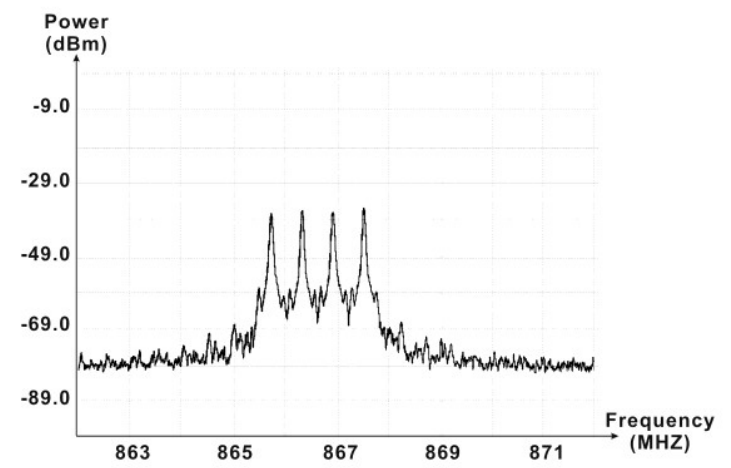

Fig. 11. Screenshot of real-time leakage suppression in four channels with updating status

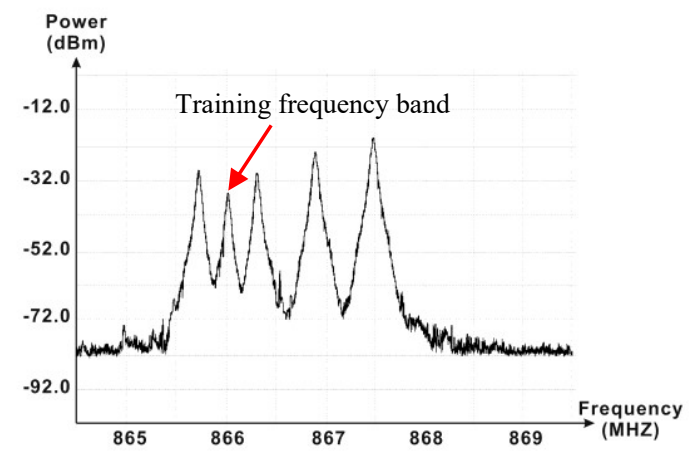

Fig. 12. Screenshot of real-time leakage suppression in four channels without updating status

However, this measurement is based on only one communication channel. In the RFID protocol, frequency hopping is required to avoid interference between different readers and to conform with radio regulations. As the hopping is a reader configuration parameter, it is difficult to set a fixed time to limit the leakage canceller scanning period. However, 
the leakage canceller can be tested under two assumptions: one is that the hopping time is long enough for the canceller to update its optimal status, and the other is that the hopping time is shorter than the canceller scanning time.

Where the hopping rate is slow compared to the LCB searching time on each frequency, the performance should be similar to the single channel result. In order to verify this assumption, the proposed RFID system hops the carrier frequency over four channels $(865.7 \mathrm{MHz}, 866.3 \mathrm{MHz}, 866.9$ $\mathrm{MHz}$, and $867.5 \mathrm{MHz}$ ). In this test, the dwell time is $1.5 \mathrm{~s}$ and the transmission power was increased to $32 \mathrm{dBm}$, and the leakage power measured in the receiver was $-0.87 \mathrm{dBm}$. After operating the leakage canceller, as expected, the leakage in four channels all reduced to around $-37.1 \mathrm{dBm}$, and the leakage suppression effect was $36.2 \mathrm{dBm}$. Fig. 11 displays the screenshot of a real-time maximum hold result. Based on this test, it approves that the leakage suppression level in four channels are similar and the assumption is correct.

If the dwell time on each channel is shorter than the canceller scanning time, the leakage canceller must be trained once on a single frequency, and its optimal parameters fixed and used on the other frequencies. In this test, the transmission power remained at $32 \mathrm{dBm}$, and the receiver encountered a leakage of $1.63 \mathrm{dBm}$. At first, the proposed leakage canceller searched its optimal status based on the training signal at 866 $\mathrm{MHz}$. It was assumed that the training frequency cannot match the hopping frequencies due to delayed updates. After the settling time, the system started to hop the operating frequency at $865.7 \mathrm{MHz}, 866.3 \mathrm{MHz}, 866.9 \mathrm{MHz}$, and 867.5 MHz; the result of which is shown in Fig. 12. As can be seen from Fig. 12 , the leakage suppression is different in each channel due to small errors in the phase and amplitude of the canceller signal. The first two channels are close to the training frequency (866 $\mathrm{MHz}$ ), and have slightly better suppression than the other channels. Even though the leakage suppression effect at 866 $\mathrm{MHz}$ can still reach $37 \mathrm{~dB}$, the suppression effect at 867.5 $\mathrm{MHz}$ is only $22 \mathrm{~dB}$. The leakage canceller is challenged when operating in such a situation. Therefore, to ensure the leakage canceller keeps updating its optimal status, the settling time becomes critical. Based on the measurement, the proposed algorithm allows the canceller to find its optimal status within

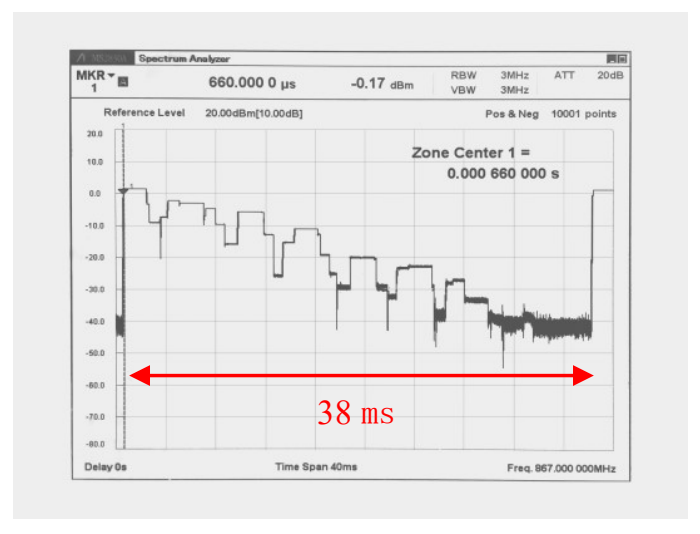

Fig. 13. Leakage suppression block scanning time

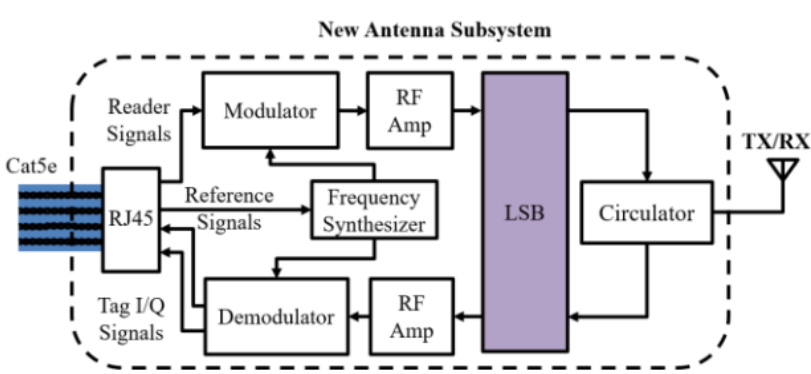

Fig. 14. Block diagram of new antenna subsystem

$38 \mathrm{~ms}$ based on 50 samples taken at each point (Fig. 13). In other similar leakage cancellation designs, the demonstrated scanning times found in [16] and [17] were $43 \mathrm{~ms}$ and $330 \mathrm{~ms}$ respectively, and their leakage cancellation level ranged from $40 \mathrm{~dB}$ to $55 \mathrm{~dB}$ respectively under different leakage levels and settling times. However, in these studies, no tag detection results are presented. Compared with these designs, the proposed LCB is sufficient for many commercial RFID readers to operate with the frequency hopping technique. Reducing the number of samples for each point or adding more advanced algorithms of phase and gain searching can further improve the canceller settling time.

Based on these experiments, this new subsystem with a leakage cancellation block can successfully solve the leakage problems. By adding a circulator before the antenna port, the system can operate in monostatic configuration (Fig. 14). The monostatic configuration can deliver $-85 \mathrm{dBm}$ sensitivity over a $300 \mathrm{~m}$ Cat5e cable. By simply doubling the magnitude of the demodulator output signals, the sensitivity of the new system can be further improved to $-88 \mathrm{dBm}$. The better sensitivity reveals the capability of this new system in improving its uplink SNR. The performance of this proposed system has proved comparable to commercial RFID systems with short coax antenna connections operating in monostatic mode.

\section{COMPARISION AND DISCUSSION}

\section{A. Passive Tag Detection}

In order to fully understand the real detection performance of this RFID system, an experimental comparison against an existing commercial off-the-shelf (COTS) RFID system was conducted. As can be seen from Fig. 15(I), a 300 m twistedpair cable is used to connect the central controller and antenna subsystem. A $6 \mathrm{dBic}$ circularly polarized antenna is directly attached to the subsystem at a height of $1.5 \mathrm{~m}$, and a target composed of 34 passive tags mounted on a board is placed at the same height approximately $2.5 \mathrm{~m}$ away from the antenna. UPM Raflatac DogBone [25] are placed in horizontal and vertical directions and distributed over a $1.8 \mathrm{~m} \times 2 \mathrm{~m}$ area. Fig. 15(II) shows that a $1 \mathrm{~m}$ coaxial cable is used to connect the COTS reader and antenna. The same antenna installed at the same height, and with the same orientation is used for both systems. The target tags were also fixed during the measurement. A transmission power of $20.5 \mathrm{dBm}$ was used in both systems, and frequency hopping over the ETSI band was 

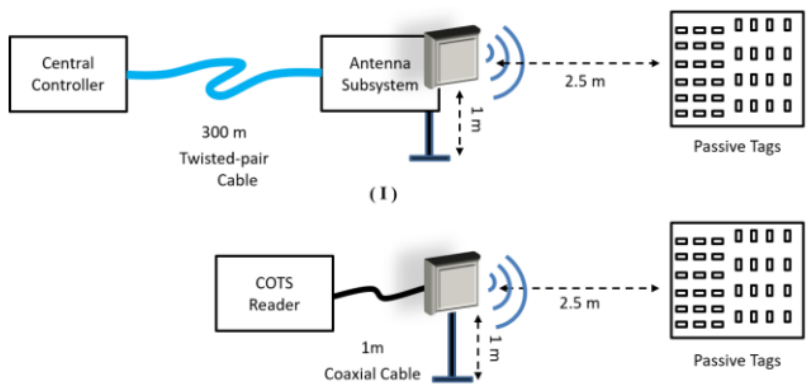

(II)

Fig. 15. Configurations of two automatic passive tag detection systems

employed. Session 0 was employed at the air interface allowing re-reading of the tags. The measurements for each system were repeated 3 times, and the average values are presented in Fig. 16. Each time the tag detection operates over 3 minutes.

According to the results (Fig. 16), the two systems provided similar detection performance. Those passive tags in green colour were successfully detected in the 3 measurements, whereas the tags in red colour failed to be detected at least once (but may have been detected on other occasions). In terms of the read rate, the proposed system can reach an average of $57.3 \mathrm{tag} / \mathrm{s}$, but the commercial one reached only $17.2 \mathrm{tags} / \mathrm{s}$. This superiority is also reflected in the time to detect all the passive tags. The designed automatic detection system was able to obtain all the tag information within 728 $\mathrm{ms}$. However, the commercial system struggled to detect some missing tags and required approximately 1.1 minutes to read them all. Further comparison of the specifications of these two systems demonstrates the better performance of the proposed system is mainly due to its superior sensitivity and better uplink SNR.

It is also worth considering the wired/wireless read range of the two systems. The results for the proposed new system can be achieved over a $300 \mathrm{~m}$ cable from the central controller, while the commercial system is much more limited in cable length. Based on the specification of LMR-200 coaxial cable, the cable loss is $0.4 \mathrm{~dB} /$ metre. For a $25 \mathrm{~m}$ long coaxial link, 10 $\mathrm{dB}$ loss will be incurred. Almost half of the signal power is wasted in the cable transmission. Although higher quality coaxial cables with lower attenuation can be used to reduce this loss, the cost of these cables and their installation is prohibitive for many RFID applications. To compensate for the cable loss, it is also possible to use additional RF amplifiers. However, this method directly leads to higher power consumption of the system. More importantly, the minimum SNR of the uplink signals is the critical parameter to allow successful decoding. Although it also can apply LNA before the cable loss, it will directly increase the antenna complexity and therefore require extra power supply for the LNA. Thus, it is difficult to improve this degradation in a high loss cable link. However, in the proposed system, a carefully designed operational amplifier can further improve the SNR of the down-converted signals before the tag signals transmit back to the controller over a Cat5e cable.

\begin{tabular}{|c|c|c|}
\hline & 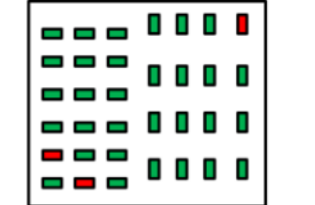 & 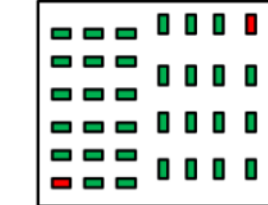 \\
\hline & $\begin{array}{l}\text { Commercial Off-the-shelf (COTS) } \\
\text { RFID System }\end{array}$ & $\begin{array}{l}\text { Proposed RFID System over } \\
300 \mathrm{~m} \text { Cat5e }\end{array}$ \\
\hline RX Sensitivity & $-84 \mathrm{dBm}$ & $-88 \mathrm{dBm}$ \\
\hline \begin{tabular}{|l|} 
Duration \\
\end{tabular} & 3 mins & $3 \mathrm{mins}$ \\
\hline $\begin{array}{l}\text { Frequency } \\
\text { Hopping }\end{array}$ & \begin{tabular}{|l}
4 channels \\
$(865.7 \mathrm{MHz}, 866.3 \mathrm{MHz}$, \\
$866.9 \mathrm{MHz}, 867.5 \mathrm{MHz})$ \\
\end{tabular} & \begin{tabular}{|l|}
4 channels \\
$(865.7 \mathrm{MHz}, 66.3 \mathrm{MHz}$ \\
$866.9 \mathrm{MHz}, 867.5 \mathrm{MHz})$ \\
\end{tabular} \\
\hline Rate & $17.16 \mathrm{tags} / \mathrm{s}$ & $57.31 \mathrm{tags} / \mathrm{s}$ \\
\hline \begin{tabular}{|l|} 
Time to see all \\
\end{tabular} & $1.1 \mathrm{~min}$ & $728 \mathrm{~ms}$ \\
\hline Nulls & 3 & 2 \\
\hline
\end{tabular}

Fig. 16. Measured results of two automatic tag detection systems

\section{B. Solutions for wide-area applications}

For covering a large area, the conventional RFID system will deploy multiple readers. Since these readers are usually controlled individually, a sophisticated software platform is required to control and analyse the received data from each reader. The control software must take each reader location into account to avoid serious reader-to-reader and reader-totag collisions. Even though the multi-port readers can directly reduce the pressure of the hardware cost and local collision probability, they still suffer from the coax cable length limits. Thus, it is difficult to install a highly-reliable large-area RFID system based on conventional readers.

When a single antenna subsystem is connected to the central controller, the blocks of an entire RFID system are similar to a conventional RFID reader and therefore the system power consumption will also be similar. For multiantenna cases, the proposed system requires less power consumption since it only needs to support multiple front-end components instead of entire RFID readers. No extra power consumption for the compensation of the cable loss is required in the new system. It may be argued that the proposed RFID system will lead to a high cost in terms of multiple front-end components in the subsystems. However, the cost of cable links, installation, and system design can be significantly reduced by using proposed system structure. This advantage becomes significant if long deployment distance is required. For example, when a larger coverage area than the maximum coverage of a conventional RFID system is required, the standard approach would need to install a whole new system including new hardware sets. However, the proposed system only needs a twisted-pair cable and a subunit.

As well as technical issues of the conventional RFID system, other practical considerations such as the layout of coaxial and power supply cable routes and deployments of devices limit the wide adoption of RFID. The labour cost of installation is significant as both Ethernet (for data) and coaxial cables must be deployed. Co-axial cables are particularly difficult to field terminate which adds to the design burden or they require excess cable to be coiled. However, the proposed 
system can overcome these challenges to an extent. In particular, the use of only Cat5e cable will reduce the installation costs as the termination is simpler, and the ability to have longer runs provides more flexibility in locating the reader hardware. A commercial implementation would integrate the remote unit with the antennas to further reduce installation requirements. The coherence between the antenna subunits will allow recent techniques for improved tag detection [7] to be applied.

For some current wired/wireless Ethernet-connected RFID readers using TCP/IP protocol to achieve wide coverage, the uncertainty of the packet delay may lead to timeout issues during the operation. Besides, RFID readers in such configuration require the rigorous design to achieve coherent broadcasting. Techniques such as frequency and phase dithering for improving the system reliability are hard to apply in conventional RFID systems. However, in the proposed RFID system, the worst case of the propagation delay in a 300 $\mathrm{m}$ Cat5e cable is less than $2 \mu \mathrm{s}$. When the backscatter link frequency is $640 \mathrm{kHz}$, the minimum time gap during the reader-tag communication is $16 \mu \mathrm{s}$. Thus, only when the Cat5e cable length reaches a kilometre, do the delay effects become serious. With aid of reference tone, the proposed sub-units can be easily synchronized.

\section{CONCLUSION}

A cost-effective and high-flexible UHF RFID system using Cat5e Ethernet cable to address remote subsystems has been demonstrated. It has been that the reader sensitivity of the preliminary system can achieve $-94.5 \mathrm{dBm}$ over $30 \mathrm{~m}$ Cat5e cable, and its sensitivity can remain at around $-94.2 \mathrm{dBm}$ when using $150 \mathrm{~m}$ Cat5e cable. By adding an effective leakage canceller block and uplink line driver, the new system can achieve $-88 \mathrm{dBm}$ reader sensitivity with an average leakage suppression of $36.9 \mathrm{~dB}$ over a $300 \mathrm{~m}$ Cat5e cable.

A comprehensive comparison study between this new system and the conventional systems currently available has been demonstrated. It shows that this new system configuration provides its high performance for long-distance passive RFID applications. At present, most models and specifications for twisted-pair cables are designed for Ethernet communication. However, communication over these twistedpair cables relating to the RFID baseband is just past the initial start-up stage. It is important now to move beyond this to achieve a full cyber-physical RFID system.

\section{REFERENCES}

[1] X. Chen, R. Huang, L, Shen, H. Chen, D. Xiong, L. Liu, C. Zhang, and R. Xu, "Design of handheld meter reading terminal based on UHF RFID," in 2nd International Workshop on Renewable Energy and Development Conference, pp. 1-7, 2018.

[2] A.M. Costin, J. Teizer, and B. Schoner, "RFID and BIM-enabled worker location tracking to support real-time building protocol and data visualization," ITcon, vol. 20, pp. 495-517, 2015.

[3] The National Institute for Occupational Safety and Health (NIOSH), Advanced Tutorial on Wireless Communication and Electronic Tracking: Electronic Tracking Systems Performance, [online]. Available from: https://www.cdc.gov/niosh/mining/content/emergencymanagementandre sponse/commtracking/advcommtrackingtutorial3.html [Access 12 2019]
[4] V.G. Buendia, S. Kenny, S.K. Podilchak, G. Goussetis, A. Costanzo, and P. Nicole, "Smart cable for Radio Frequency Indentification in aeroautical applications," in $201610^{\text {th }}$ European Conference on Antennas and Propagation, pp.1-3, April 2016.

[5] J. Rodas, V. Barral, C.J. Escudero and R. Langwieser, "A Solution for Optimizing Costs and Improving Diversity of RFID Readers," in 19th International Conference on Systems, Signals and Image Processing, pp. 84-88, April 2012.

[6] Y. Wang, N. Zhang, and L. Jin, "The Hardware Design and Implementation of Four-Channel UHF RFID Reader Based on Impinj R2000," in $20156^{\text {th }}$ IEEE International Conference on Software Engineering and Service Science, September 2015.

[7] S. Sabesan, M.J. Crisp, R.V. Penty, and I.H. White, "Wide Area Passive UHF RFID System Using Antenna Diversity Combined With Phase and Frequency Hopping," Antennas and Propagation, IEEE, vol. 62, no. 2, pp. 878-888, 2014.

[8] Indy R2000 Datasheet, [Online]. Available from: https://support.impinj.com/hc/en-us/articles/202755828-Indy-R2000

[9] SAM7 Series Summary: AT91SAM7S256 Datasheet, [Online]. Available from: http://www.atmel.com/devices/AT91SAM7S256.aspx

[10] Z. Fu, M.J. Crisp, S. Yang, R.V. Penty, and I.H. White, "Long distance passive UHF RFID system over ethernet cable," in IEEE International Conference on RFID Technology \& Application (RFID-TA), pp.294298, 2017.

[11] AD8349 Datasheet, Analog Devices, [Online]. Available from: $\mathrm{http} / / / \mathrm{www}$.analog.com/en/products/rf-microwave/iq-modulatorsdemodulators/iq-modulators/ad8349.html\#product-overview

[12] ADL5380 Datasheet, Analog Devices, [Online]. Available from: http://www.analog.com/en/products/rf-microwave/iq-modulatorsdemodulators/iq-demodulators/adl5380.html\#product-overview

[13] ADF4350 Datasheet, Analog Devices, [Online]. Available from: http://www.analog.com/en/products/rf-microwave/pll-synth/fractional-nplls/adf4350.html

[14] RF5110G Datasheet, RFDM, [Online]. Available from: http://www.qorvo.com/products/p/RF5110G\#parameters

[15] ZX60-0916LN+ Datasheet, Mini-Circuits, [Online]. Available from: https://www.minicircuits.com/WebStore/dashboard.html?model=ZX600916LN-S\%2B.

[16] K. Kapucu, M. Pauli and C. Dehollain, "A Fast Active Leakage Cancellation Method for UHF RFID Readers," in IEEE International Conference on RFID, 2017.

[17] I. Mayordomo and J. Bernhard, "Implementation of an adaptive leakage cancellation control for passive UHF RFID readers," in IEEE International Conference on RFID, 2011.

[18] D. P. Villame and J. S. Marciano, "Carrier suppression locked loop mechanism for UHF RFID readers," in IEEE International Conference on RFID, Orlando, 2010.

[19] AD8340 Datasheet, Analog Devices, [Online]. Available from: https://www.analog.com/en/products/ad8340.html\#.

[20] ADL5513 Datasheet, Analog Devices, [Online]. Available from: https://www.analog.com/en/products/adl5513.html.

[21] PIC16F1779 Datasheet, MICROCHIP, [Online]. Available from: https://www.microchip.com/wwwproducts/en/PIC16F1779.

[22] LMR-400 Datasheet, Times Microwave, [Online]. Available from: https://www.timesmicrowave.com/documents/resources/LMR-400.pdf

[23] LMR-1700 Datasheet, Times Microwave, [Online]. Available from: http://www.timesmicrowave.com/documents/resources/LMR-1700.pdf

[24] TC-2600A Datasheet, [Online]. Available from; https://mcstestequipment.com/product/tescom-tc2600a-rfid-test-system/

[25] UPM Raflatax DogBone Datasheet, [Online]. Available from: http://www.fastrfid.com/raflatac/UHF/tech_speck_3001572_letter.pdf.

[26] T. Standard, "TIA-568-C2 Standard," TIA, 2009. [Online]. Available: http://innovave.com/wp-content/uploads/2016/01/TIA-568-C.2.pdf. [Accessed 09 2018]. 
